\title{
PSEUDOMONAS VACCINE AND HYPERIMMUNE PLASMA \\ IN THE TREATMENT OF THE SEVERELY BURNED PATIENT
}

\section{(A PROGRESS REPORT)}

\author{
Irving Feller \\ Burn Unit \\ University of Michigan Medical Center \\ Ann Arbor, Mich.
}

Septicemia due to gram-negative bacteria is a serious problem for all debilitated patients, ${ }^{1}$ and especially those with severe burns. Pseudomonas aeruginosa emerged as the most common organism found in burned patients who died from septicemia. ${ }^{2}$ This presentation reviews the laboratory work and clinical trials with both pseudomonas vaccine and hyperimmune plasma in the treatment of the severely burned patient and brings up to date the results previously reported. ${ }^{3}$

It is necessary to define the severe burn, since patients with small and moderate burns are usually not troubled by gram-negative septicemia. The severe burn in this report is defined as one involving a minimum of twenty percent full-thickness skin loss (third degree) or forty percent total body burn (second and third degree).

A series of rabbit experiments designed to determine the antibody response to active immunization with the injection of a pseudomonas vaccine were completed five years ago. ${ }^{4}$ An antibody response was obtained in four weeks, and the six-pound rabbits were then found to have eighty percent protection against i.v. injection of three billion live organisms, compared with a control group which did not receive the vaccine. A second set of rabbit experiments was conducted to determine the value of hyperimmune plasma for passive immunity. Again, a satisfactory protection was obtained by giving the hyperimmune plasma before and after the i.v. challenge of the live organisms. The vaccine was prepared for normal human volunteers. When tested, it was found that a longer time was required to develop a titer in the human than in the rabbit, but a good antibody level could be achieved. There were no adverse reactions to the vaccine in the human volunteers and a hyperimmune plasma bank was established. The volunteers are now maintained at a high titer by interval vaccinations and their plasma is collected, frozen and stored. A daily program was started in which all adults with severe burns received $250 \mathrm{ml}$ of the hyperimmune plasma while the children were given $125 \mathrm{ml}$.

From July, 1959, to July 1966,126 patients with burns greater than twenty percent being full-thickness, or wounds involving forty percent of the total body surface, were admitted to the University of Michigan Burn Unit. Patients who died in less than six days of causes other than infection are not included. All deaths after six days are included. TABLE 1 shows three different groups of patients during the six-year-period. It is understood that this is not an ideal method of comparing patients, but it does represent a trend in results, inasmuch as the same pattern of therapy is carried out today that was instituted in our Burn Unit six years ago. The use of hyperimmune plasma is a major change in infection control, while antibiotic therapy and dressing-change technique have not changed.

The first group consisting of 41 patients, were treated prior to the use of the vaccine. The second group, of 24 patients, received the vaccine alone and not the hyperimmune plasma. The third group, of 61 patients, received the hyper- 
TABLE 1

Pseudomonas Vaccine and Hyperimmune Plasma in Burn Patient Treatment*

\begin{tabular}{|c|c|c|c|}
\hline & $\begin{array}{c}\text { Not } \\
\text { Vaccinated } \\
1 . \\
1959-62\end{array}$ & $\begin{array}{c}\text { Vaccinated } \\
\text { Only } \\
\text { III. } \\
1962-63\end{array}$ & $\begin{array}{c}\text { Vaccinated } \\
\text { Hyperimmune } \\
\text { Plasma } \\
\text { III } \\
\text { 1963-7/66 }\end{array}$ \\
\hline No. of Patients & 41 & 24 & 61 \\
\hline Av. Age (Yrs.) & 26 & 24 & 24 \\
\hline Av. \% Total burns & 51 & 40 & 44 \\
\hline Av. \% Full-thickness burns & 38 & 31 & 30 \\
\hline \multicolumn{4}{|l|}{ Deaths } \\
\hline No. of deaths & 19 & 9 & 11 \\
\hline$\%$ Mortality & 44 & 38 & 18 \\
\hline Av. age (Yrs.) & 34 & 26 & 43 \\
\hline Av. \% Full-thickness burns & 46 & 40 & 52 \\
\hline \multicolumn{4}{|l|}{ Septicemias } \\
\hline Total No. & 29 & 18 & 16 \\
\hline$\%$ Septicemias (all patients) & 71 & 75 & 26 \\
\hline No. Septicemia deaths & 17 & 9 & 6 \\
\hline \% Septicemia deaths of all deaths & 88 & 100 & 55 \\
\hline No. Pseudomonas septicemias & 21 & 7 & 3 \\
\hline \% Pseudomonas septicemias (all patients) & 51 & 29 & 5 \\
\hline \% Pseudomonas septicemias of all septicemias & 72 & 39 & 19 \\
\hline No. Pseudomonas deaths & 13 & 6 & 2 \\
\hline \% Pseudomonas deaths, all patients & 32 & 25 & 3 \\
\hline
\end{tabular}

* Summary of 126 cases with burns greater than $20 \%$ full-thickness or $40 \%$ total body surface, excluding deaths within six days of burn.

immune plasma daily, as well as the weekly injection of the vaccine to help stimulate their own antibodies. The average age, percent total burn and percent full-thickness burn is given for each of the three groups, and it is apparent that as groups the patients are of comparable age and injury.

There were 19 deaths in the first group; nine in the second and eleven in the third. The average ages of the patients who died were 34,26 and 43 years, respectively. The average percent of full-thickness burn in the first group of deaths was 46 percent; in the second group, 40 percent; and in the third group, 52 percent. This illustrates that the patients in group III had more severe burns than the patients in groups I and II.

A review of the septicemia problem reveals that group I had 29 cases of septicemia out of a total of 41 patients. In group II, there were 18 cases of septicemia out of 24 patients, and in the largest group, 61 patients in group III, the incidence of septicemia dropped to its lowest point of 16 . There were 17 deaths in group I, nine in group II and six in group III. There were 21 cases of pseudomonas septicemia in the first group; seven in the second; and three in the last. There were only two pseudomonas deaths in group III in contrast to 13 in group I.

Results of this program have been encouraging to date and a continued program of evaluation is in progress.

\section{SUMMARY}

This progress report demonstrates a pseudomonas vaccine and hyperimmune plasma used in treating 61 patients with burns involving a minimum of 20 percent 
Feller: Pseudomonas Vaccine \& Hyperimmune Plasma 1003

of the body with full-thickness (third degree) loss of 40 percent total injury (partial thickness and full-thickness). The incidence of septicemia had decreased and mortality due to pseudomonas septicemia, when it does occur, has been greatly reduced.

\section{References}

1. Feller, I. 1965. Changes in the etiology of septicemia. In Control of Infections in Hospitals. Univ. of Mich. Publication. : 39-43.

2. Feller, I. \& R. C. HendriX. 1964. Clinical pathologic study of sixty fatally burned patients. Surg. Gyn. \& Obs. 119: 1-5.

3. Feller, I. \& I. Kamfi. 1964. Pseudomonas vaccine and hyperimmune plasma in the treatment of burned patients. (Interval report) Amer. Coll. of Surg. Forum. : Vol. 15.

4. Feller, I., A. B. Vial, W. S. Collahen \& J. Waldyke. 1964. Use of vaccine and hyperimmune serum for protection against pseudomonas septicemia. J. of Trauma 4: 451-456. 